\title{
Magnetically driven loss of centrosymmetry in metallic Pb2CoOsO6
}

\author{
A. J. Princep, H. L. Feng, Y. F. Guo, F. Lang, H. M. Weng, P. \\ Manuel, D. Khalyavin, A. Senyshyn, M. C. Rahn, Y. H. Yuan, \\ Y. Matsushita, S. J. Blundell, K. Yamaura, and A. T. \\ Boothroyd
}

\section{Published version information}

Citation: AJ Princep et al. "Magnetically driven loss of centrosymmetry in metallic Pb2CoOsO6." Phys Rev B 102, no. 10 (2020): 104410.

DOI: $10.1103 /$ PhysRevB.102.104410

This version is made available in accordance with publisher policies. Please cite only the published version using the reference above. This is the citation assigned by the publisher at the time of issuing the APV. Please check the publisher's website for any updates. 


\title{
Magnetically driven loss of centrosymmetry in metallic $\mathrm{Pb}_{2} \mathrm{CoOsO}_{6}$
}

\author{
A. J. Princep, ${ }^{1,2,{ }^{*}}$ H. L. Feng, ${ }^{3,4, \dagger}$ Y. F. Guo, ${ }^{5,}$ F. Lang, ${ }^{1}$ H. M. Weng, ${ }^{4,6}$ P. Manuel, ${ }^{2}$ D. Khalyavin, ${ }^{2}$ A. Senyshyn, ${ }^{7}$ \\ M. C. Rahn, ${ }^{1,8}$ Y. H. Yuan, ${ }^{3}$ Y. Matsushita, ${ }^{3}$ S. J. Blundell, ${ }^{1}$ K. Yamaura, ${ }^{9,},{ }^{8}$ and A. T. Boothroyd ${ }^{1, \|}$ \\ ${ }^{1}$ Department of Physics, University of Oxford, Clarendon Laboratory, Parks Road, Oxford OX1 3PU, United Kingdom \\ ${ }^{2}$ ISIS Facility, Rutherford Appleton Laboratory, Chilton, Didcot OX11 OQX, United Kingdom \\ ${ }^{3}$ National Institute for Materials Science, 1-1 Namiki, Tsukuba, Ibaraki 305-0044, Japan \\ ${ }^{4}$ Beijing National Laboratory for Condensed Matter Physics, Institute of Physics, Chinese Academy of Sciences, Beijing 100190, China \\ ${ }^{5}$ School of Physical Science and Technology, ShanghaiTech University, Shanghai 201210, China \\ ${ }^{6}$ Collaborative Innovation Center of Quantum Matter, Beijing 100190, China \\ ${ }^{7}$ FRM-II, Technische Universität München, Garching 85747, Germany \\ ${ }^{8}$ Institute for Solid State and Materials Physics, Technical University of Dresden, 01062 Dresden,Germany \\ ${ }^{9}$ International Center for Materials Nanoarchitectonics (WPI-MANA), National Institute for Materials Science, \\ 1-1 Namiki, Tsukuba, Ibaraki 305-0044, Japan
}

(Received 13 February 2019; revised 22 July 2020; accepted 30 July 2020; published 8 September 2020)

\begin{abstract}
We report magnetic, transport, neutron diffraction, and muon spin rotation data showing that $\mathrm{Pb}_{2} \mathrm{CoOsO}_{6}$, a metallic double-perovskite with a centrosymmetric space group at room temperature exhibits a continuous second-order phase transition at $45 \mathrm{~K}$ to a magnetically ordered state with a noncentrosymmetric space group. The absence of inversion symmetry is very uncommon in metals, particularly metallic oxides. In contrast to the recently reported ferroelectriclike structural transition in $\mathrm{LiOsO}_{3}$, the phase transition in $\mathrm{Pb}_{2} \mathrm{CoOsO}_{6}$ is driven by a long-range collinear antiferromagnetic order, with propagation vector $\mathbf{k}=\left(\frac{1}{2}, 0, \frac{1}{2}\right)$, which relieves the frustration associated with the symmetry of the magnetic exchanges. This magnetically driven loss of inversion symmetry represents another frontier in the search for novel metallic behavior.
\end{abstract}

DOI: 10.1103/PhysRevB.102.104410

\section{INTRODUCTION}

Metals whose crystal structure lacks a center of inversion symmetry have been attracting increasing interest owing to the phenomena they can exhibit, such as optical activity [1] and a highly anisotropic thermopower, (a desirable property of certain thermoelectric devices) [2]. Noncentrosymmetric metals having strong electronic correlations can support exotic emergent quasiparticles, including skyrmions in chiral magnets [3]. Noncentrosymmetric superconductors are of particular interest because they can have spin-polarized supercurrents and unconventional pairing states with mixed singlet-triplet character even in the absence of strong electronic correlations [4-6]. The key feature of noncentrosymmetric systems is a band splitting throughout much of momentum space caused by spin-orbit coupling (Dresselhaus splitting [7]) which leads to a nontrivial topology of the electronic wave functions and plays an essential role in all these phenomena.

Noncentrosymmetric metals (NCSMs) are relatively uncommon. This is because conduction electrons can effectively screen the electric dipole formation which is generally associated with acentricity. In fact, there exist to date only around 30 known NCSMs, of which only a handful are metallic

\footnotetext{
*princep@physics.ox.ac.uk

†hai.feng@iphy.ac.cn

‡guoyf@shanghaitech.edu.cn

\$Yamaura.kazunari@nims.go.jp

"a.boothroyd@physics.ox.ac.uk
}

oxides [2]. Nevertheless, oxides are particularly attractive for device applications due to their stability under typical operating conditions, so the discovery of more oxide NCSM would be of considerable interest.

Structural transitions in metallic oxides which remove the center of inversion symmetry have previously been observed in $\mathrm{Cd}_{2} \mathrm{Re}_{2} \mathrm{O}_{7}$ [8] and $\mathrm{LiOsO}_{3}$ [9]. The origin and nature of the transition in $\mathrm{Cd}_{2} \mathrm{Re}_{2} \mathrm{O}_{7}$ is uncertain, but $\mathrm{LiOsO}_{3}$ was found to be an example of what Anderson and Blount referred to as a "ferroelectric metal", i.e., a metal having a continuous structural phase transition accompanied by the appearance of a polar axis and the disappearance of an inversion center [10]. The phase transition in $\mathrm{LiOsO}_{3}$ is driven by the ordering of $\mathrm{Li}$ ion displacements [9].

Here, we report structural, magnetic and transport measurements of $\mathrm{Pb}_{2} \mathrm{CoOsO}_{6}$, another metal that undergoes a phase transition to a noncentrosymmetric structure. In this case, however, we show that the loss of inversion symmetry is driven by magnetoelastic coupling to a pair of antiferromagnetic order parameters which relieve the magnetic frustration of the higher symmetry phase, analogous to the behavior of a type-II hybrid-improper multiferroic [11,12]. This represents a paradigm for obtaining NCSM using magnetic frustration as a key ingredient.

\section{STRUCTURAL AND BULK CHARACTERIZATION}

Polycrystalline and single crystal $\mathrm{Pb}_{2} \mathrm{CoOsO}_{6}$ was prepared by a high-pressure method in a belt-type 


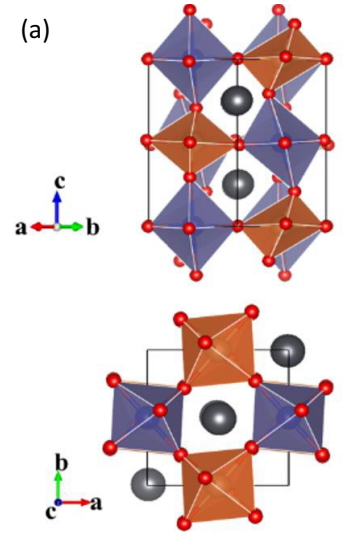

(b)
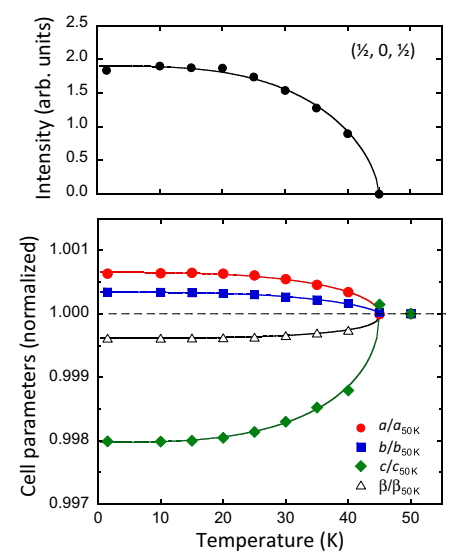

FIG. 1. (a) Crystallographic views of $\mathrm{Pb}_{2} \mathrm{CoOsO}_{6}$ along the [110] (left) and [001] (right) directions. The blue and brown octahedra represent $\mathrm{CoO}_{6}$ and $\mathrm{OsO}_{6}$, respectively. The large grey solid spheres represent $\mathrm{Pb}$. (b) Upper: Temperature dependence of the fundamental magnetic reflection measured in neutron scattering. Lower: Temperature dependence of the lattice parameters below the ordering temperature, relative to their value at $T_{\mathrm{N}}$. Solid lines are a guide to the eye.

high-pressure apparatus [13,14] (see Supplemental Material [15]). The room temperature crystal structure of polycrystalline $\mathrm{Pb}_{2} \mathrm{CoOsO}_{6}$ was initially determined by synchrotron $\mathrm{x}$-ray diffraction (XRD) using the large Debye-Scherrer camera at the BL15XU beamline in SPring-8, Japan [16]. The obtained SXRD patterns were analyzed by the Rietveld method using the programs RIETAN-2000 [17] and VESTA [18] (see Supplemental Material [15]). The structure found is the fully ordered double perovskite structure with space group of $\mathrm{P} 2{ }_{1} / n$ (tilt pattern $\mathrm{a}^{-} \mathrm{a}^{-} \mathrm{c}^{+}$in the notation of Glazer [19]). The Co and Os atoms fully occupy the Wyckoff positions $2 a$ and $2 b$, respectively, with no site mixing observed to the accuracy of the measurement. The bond valence sums imply +2 and +6 valence states for the Co and Os atoms, respectively [20,21]. The refined crystal structure is depicted in Fig. 1(a). The degree of distortion from the cubic structure is indicated by the deviation of interoctahedral $\mathrm{Co}-\mathrm{O}-\mathrm{O}$ s bond angles which are $180^{\circ}$ in the ideal structure, but here we find them to be $168^{\circ}, 172^{\circ}$, and $145^{\circ}$, respectively, implying substantial buckling of the octahedral connections.

The temperature variation of the structure of $\mathrm{Pb}_{2} \mathrm{CoOsO}_{6}$ was investigated between $1.5 \mathrm{~K}$ and $300 \mathrm{~K}$ by neutron powder diffraction (NPD). The NPD measurements were carried out on the SPODI high-resolution diffractometer [22] at the FRMII reactor facility of the Technische Universität München, Germany, and also on the WISH time-of-flight diffractometer [23] at the ISIS Neutron and Muon Source, UK. The Rietveld refinement of the crystal and magnetic structures was performed using FULLPROF [24]. Rietveld refinements were carried out with the FULLPROF suite [24] using the magnetic form factor for $\mathrm{Os}^{6+}$ determined by Kobayashi et al. [25]. Results of refinement against the NPD pattern at $300 \mathrm{~K}$ are fully consistent with the synchrotron XRD results, including the absence of any $\mathrm{Co} / \mathrm{Os}$ site mixing to within the experimental uncertainty of $\approx 1 \%$. The lattice parameters

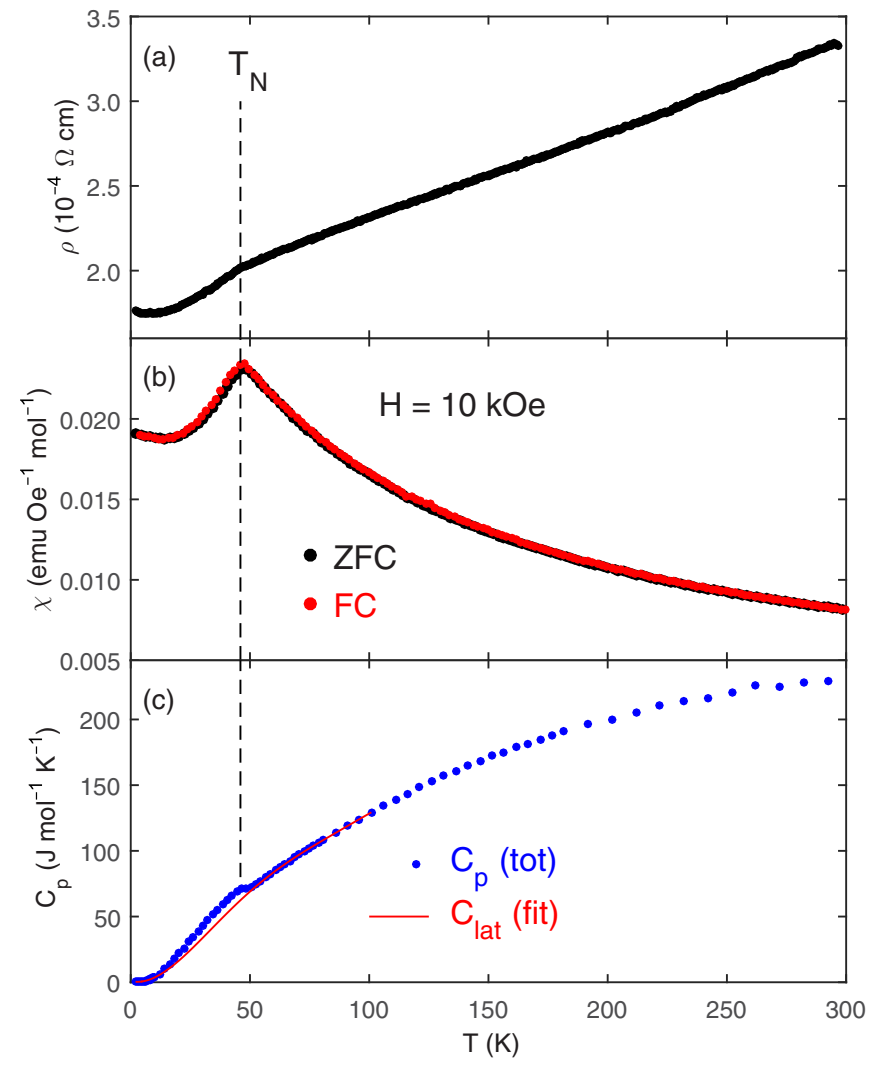

FIG. 2. Temperature dependence of bulk properties of $\mathrm{Pb}_{2} \mathrm{CoOsO}_{6}$. (a) Resistivity $\rho$ of a single crystal. (b) Magnetic susceptibility $\chi$ of the polycrystalline sample. (c) Heat capacity $C_{\mathrm{p}}$ of the polycrystalline sample.

decrease monotonically with temperature below $300 \mathrm{~K}$ until $T_{\mathrm{N}}=45 \mathrm{~K}$, below which the parameters $a$ and $b$ increase slightly while $c$ decreases significantly for a small net reduction in the unit-cell volume [see Fig. 1(b)]. This abrupt change in behavior of the lattice parameters is typically indicative of a magnetoelastic structural distortion accompanying the magnetic order, as discussed below. We identify $T_{\mathrm{N}}$ with a bulk antiferromagnetic ordering transition, based on the neutron diffraction and muon spin rotation experiments presented below, as well as by the existence of characteristic signatures in other physical properties (see Fig. 2).

Figure 2(a) presents the temperature dependence of the electrical resistivity $\rho$ of $\mathrm{Pb}_{2} \mathrm{CoOsO}_{6}$ measured on a single crystal between $2 \mathrm{~K}$ and $300 \mathrm{~K}$. The single crystal was confirmed to have the same crystal structure as the powder sample by single crystal XRD (see Supplemental Material [15]). Above $T_{\mathrm{N}}, \rho$ is roughly linear in $T$, and displays a pronounced change at $T_{N}$ without fundamentally altering its metallic character. The drop in $\rho$ below $T_{\mathrm{N}}$ is likely due to the reduction in scattering of the conduction electrons by paramagnetic fluctuations as the system undergoes magnetic ordering. At the lowest temperatures, there is a small upturn in the resistivity, however, given the resistivity decreases monotonically over several decades in temperature and there is no evidence for a further transition, we attribute this to a simple experimental artifact arising from the contacts with the sample. The DC magnetic susceptibility ( $\chi$ vs $T$ ) of 
TABLE I. Refined values of the crystal and magnetic structures at $1.5 \mathrm{~K}$ from neutron powder diffraction. Space group $\mathrm{P} 2_{1} / n$ (No. 14 , origin choice 2) $a=5.63651(1) \AA, b=5.58361(7) \AA, c=$ 7.82321(1) $\AA$, and $\beta=89.815(2)^{\circ}$. Magnetic moments on Co and Os sites were constrained to be equal. Final $R$ values are $R_{\text {nuc }}=$ $2.29 \%$ and $R_{\mathrm{mag}}=7.91 \%$. The lattice vectors of the Shubnikov group in the standard setting $\mathrm{P}_{a} c$ are related to the high temperature crystallographic space group $\mathrm{P} 21 / n$ as $(-2,0,0),(0,-1,0),(1,0,1)$ with an origin shift of $\left(0, \frac{1}{4}, 0\right)$.

\begin{tabular}{lccccc}
\hline \hline Site & Wyck & $x$ & $y$ & $z$ & $B_{\text {iso }}\left(\AA^{2}\right)$ \\
\hline $\mathrm{Pb}$ & $4 e$ & $0.0059(3)$ & $0.5127(4)$ & $0.2504(3)$ & $0.45(4)$ \\
$\mathrm{Co}$ & $2 a$ & 0 & 0 & 0 & $0.09(17)$ \\
$\mathrm{Os}$ & $2 b$ & 0 & 0 & 0.5 & $0.18(6)$ \\
$\mathrm{O} 1$ & $4 e$ & $-0.0679(4)$ & $-0.0088(7)$ & $0.2592(4)$ & $0.53(6)$ \\
$\mathrm{O} 2$ & $4 e$ & $0.2413(6)$ & $0.2810(7)$ & $0.0362(8)$ & $0.55(12)$ \\
$\mathrm{O} 3$ & $4 e$ & $0.2834(6)$ & $0.7651(6)$ & $0.0365(8)$ & $0.50(12)$ \\
$\mathrm{Site}$ & & $\mathrm{M}_{a}$ & $\mathrm{M}_{b}$ & $\mathrm{M}_{c}$ & irrep \\
\hline $\mathrm{Co}$ & & $-0.77(1)$ & 0 & $1.89(1)$ & $\mathrm{mY}_{1}^{+}$ \\
$\mathrm{Os}$ & & $-0.77(1)$ & 0 & $1.89(1)$ & $\mathrm{mY}_{2}^{-}$ \\
\hline \hline
\end{tabular}

$\mathrm{Pb}_{2} \mathrm{CoOsO}_{6}$, presented in Fig. 2(b), exhibits a sharp peak at $T_{\mathrm{N}}$, implying that the transition has a magnetic origin. A Curie-Weiss fit to $\chi^{-1}$ in the paramagnetic region above $200 \mathrm{~K}$ (see Fig. S3a in Supplemental Material ) yields the Weiss temperature $\Theta_{\mathrm{W}} \approx-106 \mathrm{~K}$, suggesting dominant antiferromagnetic $(\mathrm{AFM})$ interactions and $\left|\Theta_{\mathrm{W}} / T_{\mathrm{N}}\right| \approx 2.2$, indicative of weak magnetic frustration. The effective magnetic moment $\left(\mu_{\text {eff }}\right)$ per formula unit is approximately $4.9 \mu_{\mathrm{B}}$.

\section{MAGNETIC STRUCTURE REFINEMENT}

Below $T_{\mathrm{N}}$, additional peaks were observed in NPD patterns that could be indexed with a propagation vector $\mathbf{k}=\left(\frac{1}{2}, 0, \frac{1}{2}\right)$ [see Fig. 1(b) upper and Fig. 3(a)]. There are four magnetic irreducible representations (irreps) that are compatible with $k=\left(\frac{1}{2}, 0, \frac{1}{2}\right)$ in the space group $\mathrm{P} 2_{1} / n$ and these are the one-dimensional irreps $\mathrm{mY}_{1}^{+}, \mathrm{mY}_{2}^{+}, \mathrm{mY}_{1}^{-}$, and $\mathrm{mY}_{2}^{-}$[26]. The +-type irreps correspond to order on only the Co sites, and the - irreps to order on only the Os sites, and each irrep has a specific relation between the direction of the moments on the two equivalent metal sites. The allowed magnetic Bragg reflections vary with the choice of irrep and inspection of the NPD data allowed us to constrain the possible irreps to $\mathrm{mY}_{1}^{+}$ and $\mathrm{mY}_{2}^{-}$. A model combining these two irreps was refined against NPD data from banks 2-9 of WISH. Figure 3(a) shows data from banks 2 and 9 recorded at $1.5 \mathrm{~K}$ together with the refinement. The results of the refinement are tabulated in Table I, and the magnetic structure is shown in Fig. 3(b). A direct observation of the loss of centrosymmetry by measurement of optical second-harmonic generation or Friedel pairs would be unlikely to be fruitful, as the size of the distortion is likely to be not larger than that in magnetically induced (type-II) multiferroics where a similar mechanism pertains. There, the size of the polar distortion is of order $10 \mathrm{fm}$ (i.e., the dimensions of the atomic nucleus) and thus far too small to detect by standard methods [27].
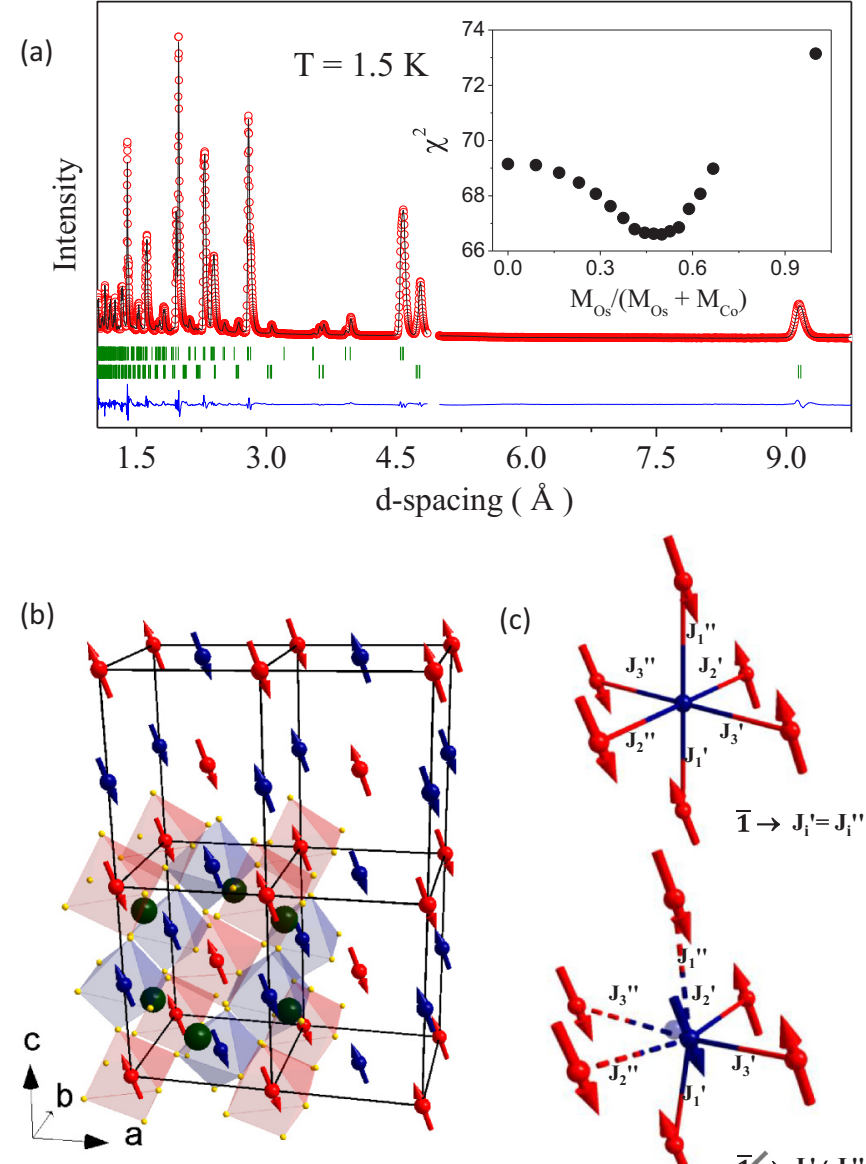

(c)

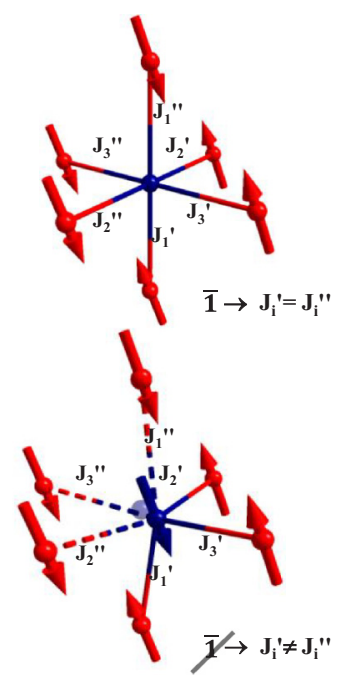

FIG. 3. Magnetic structure of $\mathrm{Pb}_{2} \mathrm{CoOsO}_{6}$. (a) Neutron diffraction data recorded in banks 2 and 9 of the WISH diffractometer are shown together with the Rietveld fit. The data are in red, the fit in black, and the difference in blue. The green tick marks indicate structural (upper) and magnetic (lower) Bragg peaks. The inset shows how the goodness-of-fit statistic $\chi^{2}$ for the Rietveld fit varies as a function of the fraction of the total ordered moment that is located on the Os site. (b) Refined magnetic structure $\left(T<T_{\mathrm{N}}\right)$, with red and blue arrows as the $\mathrm{Co}^{2+}$ and $\mathrm{Os}^{6+}$ moments, respectively. The red and blue octahedra represent the oxygen coordination polyhedra and the dark green spheres depict nonmagnetic $\mathrm{Pb}^{2+}$. (c) Absent a structural distortion, the mean field exerted by nearest neighbor atoms cancels by symmetry. Removing the center of inversion symmetry by displacing the Co and Os sublattices relative to one-another permits a non-zero nearest neighbor coupling.

The parameters describing the Co and Os moments are strongly correlated in the refinement and constraints are needed to reach convergence. This is specific to $\mathbf{k}=\left(\frac{1}{2}, 0, \frac{1}{2}\right)$ in space group $\mathrm{P} 2_{1} / n$ where both magnetic species contribute intensity to the reflections in the exact same way except for their different form factors. Initial refinements performed with moments on only the Co sites or only the Os sites indicated that the magnetic structure is collinear and that the component along the $b$ axis $\left(M_{b}\right)$ is undetectably small. Thereafter, we set $M_{b}$ to zero and performed fits as a function of the ratio of the moments on the Co and Os sites assuming all moments to be collinear. The substantial difference between the magnetic form factors of $\mathrm{Co}^{2+}$ and $\mathrm{Os}^{6+}$ provides a degree of sensitivity 
to the magnetic moment on each sublattice and the best fit was found with approximately the same moment $\left(2.04 \mu_{\mathrm{B}}\right)$ on both $\mathrm{Co}$ and Os sites inclined at an angle of about $22^{\circ}$ to the $c$ axis. Figure 3(a) shows data from banks 2 and 9 recorded at $1.5 \mathrm{~K}$ together with the refinement and the insert shows the fit quality as the Os moment fraction is varied. The results of the refinement are tabulated in Table I, and the magnetic structure is shown in Fig. 3(b).

The observation of a single magnetic transition indicates coincident order of the two sublattices, as observed recently in the osmate double-perovskite $\mathrm{Sr}_{2} \mathrm{FeOsO}_{6}$ [28]. Coincident order of coupled sublattices is to be expected unless the $\mathrm{Co}-\mathrm{O}-\mathrm{Os}$ superexchange was either highly frustrated or suppressed, in which case one expects two distinct magnetic transitions as appears to be the case in $\mathrm{Sr}_{2} \mathrm{CoOsO}_{6}$ $[29,30]$. It is worth nothing that the conclusions of the preceding symmetry analysis are not contingent on a single magnetic phase transition. If in reality one of the magnetic sublattices orders at some temperature $T_{\mathrm{N}^{\prime}}$ just below $T_{\mathrm{N}}$, the resulting state would still be without a center of symmetry and thus a magnetically driven polar metal.

As noted earlier, below $T_{\mathrm{N}}$ the temperature dependence of the lattice parameters abruptly changes and they display an order-parameter-like behavior, suggesting that the lattice distortion is coupled to the magnetic order parameter. The magnetic structure refinement and electronic-structure calculations (see below) provide evidence that there are ordered moments on both the Co and Os sites and, moreover, the refined magnetic structure does not possess a center of symmetry. Any nonzero magneto-elastic coupling will then remove the center of symmetry of the crystal structure, Fig. 3(c), relieving frustration and reducing the space group-symmetry of the structure to the (noncentrosymmetric) polar group Pn [31], with the corresponding Shubnikov group for the magnetic structure being $\mathrm{P}_{a} \mathrm{c}$. Both $\mathrm{Co}$ and $\mathrm{Os}$ have a nonzero orbital angular momentum, and so such coupling is expected to be non-negligible and is indicated by the temperaturedependent variation of the lattice parameters described earlier [see Fig. 1(a)].

This scenario is opposite to the case where relieving orbital degeneracy results in a structural phase transition (i.e., a JahnTeller transition) for which any eventual magnetic ordering is expected to occur at a lower temperatures [32]. Instead, the onset of magnetic order, via spin-orbit coupling, enforces some preferred orbital occupation which then results in a structural distortion [32] as is the case, for example, at $T_{\mathrm{N} 1}$ in $\mathrm{Sr}_{2} \mathrm{CoOsO}_{6}[29,30]$. Although clear changes in structural elements of the diffraction pattern were observed on cooling through $T_{\mathrm{N}}$, it proved impossible to refine a detailed model for the low temperature crystal structure owing to instabilities that resulted from the large number of free parameters.

\section{ELECTRONIC-STRUCTURE CALCULATIONS}

The electronic structure of $\mathrm{Pb}_{2} \mathrm{CoOsO}_{6}$ was investigated by performing first-principles calculations with the OPENMX software package [33] using the lattice parameters and the AFM configuration obtained from the NPD refinement. The choice of pseudo-potentials, basis sets, and the sampling of Brillouin zone with $6 \times 6 \times 10$ grid have been carefully

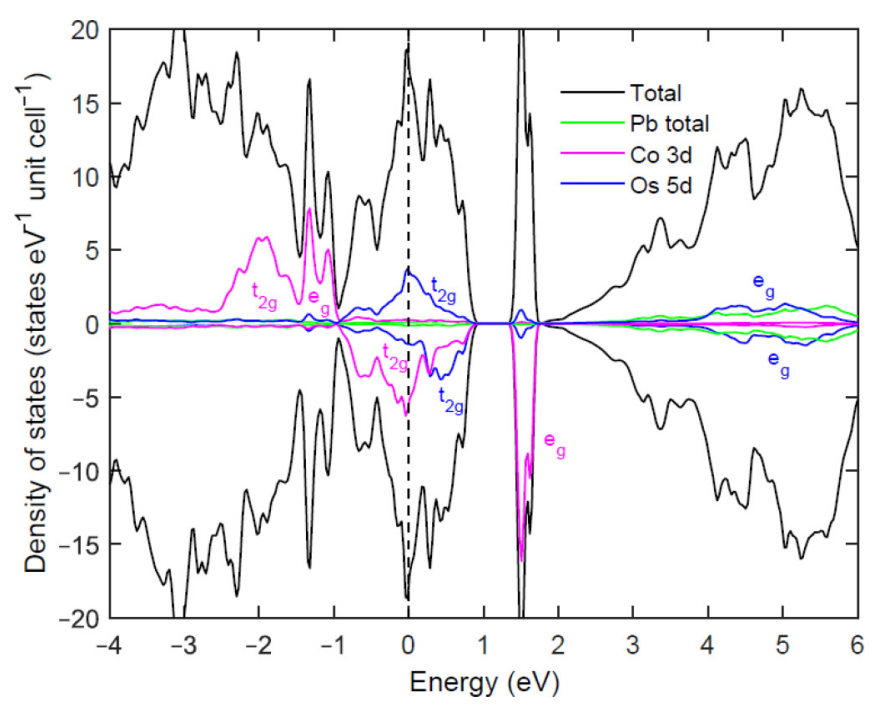

FIG. 4. Total and projected spin-polarized partial density of states (DOS) for antiferromagnetic $\mathrm{Pb}_{2} \mathrm{CoOsO}_{6}$ calculated in the GGA. Dotted (magenta) lines are for the Co $3 d$ orbitals and dashed (blue) lines are the Os $5 d$ orbitals. Those contributions from the crystal field split $\mathrm{t}_{2 g}$ and $\mathrm{e}_{g}$ orbitals are also labeled. The Fermi energy is set at zero. Positive and negative values of the DOS represent the spin-up and spin-down parts, respectively.

optimized and the exchange-correlation functional within the generalized gradient approximation (GGA) [34] was used. For Co $3 d$ orbitals, the spin splitting is larger than that due to the crystal field $(\mathrm{CF})$, so $\mathrm{Co}^{2+}$ is in its high spin state with configuration as $\mathrm{t}_{2 g}^{3 \uparrow} \mathrm{e}_{g}^{2 \uparrow} \mathrm{t}_{2 g}^{2 \downarrow} \mathrm{e}_{g}^{0 \downarrow}$, where the superscript number represents the number of occupied electrons and arrows indicate the spin state. On the contrary, the extended $5 d$ orbitals of Os have a larger CF splitting than spin splitting, which consequently results in a low spin state of $\mathrm{Os}^{6+}\left(5 d^{2}\right)$ with an approximate configuration of $\mathrm{t}_{2 g}^{1 \uparrow} \mathrm{e}_{g}^{0 \uparrow} \mathrm{t}_{2 g}^{1 \downarrow} \mathrm{e}_{g}^{0 \downarrow}$. The calculated density of states (DOS) indicates substantial contributions from both Co and Os $d$-electrons at the Fermi level, both of which are split into $\mathrm{t}_{2 g}$ and $\mathrm{e}_{g}$ manifolds under the approximately octahedral CF. This large DOS at the Fermi level is consistent with the robust metallicity we observe experimentally. The calculated local magnetic moments inferred from this are $2.46 \mu_{\mathrm{B}}$ and $0.450 \mu_{\mathrm{B}}$, on Co and Os, respectively, consistent with the above approximate atomic configuration and with the range of possible experimental values indicated by NPD [see inset to Fig. 3(a)].

\section{MUON SPIN ROTATION AND DFT $+\mu$}

The scenario of the magnetically induced structural distortion described above requires magnetic order on both the Co and Os sublattices. As a further test of this picture, we turned to zero-field (ZF) muon-spin rotation $\left(\mu^{+} \mathrm{SR}\right)$, which is a local probe of magnetism. ZF muon spin relaxation $\left(\mu^{+} \mathrm{SR}\right)$ spectra of $\mathrm{Pb}_{2} \mathrm{CoOsO}_{6}$ were measured in a ${ }^{3} \mathrm{He}$ cryostat in the general purpose spectrometer at the Swiss Muon Source at the Paul Scherrer Institute, Switzerland. The muon spectra below the transition can be well modeled (Fig. 5, blue curve) with the 


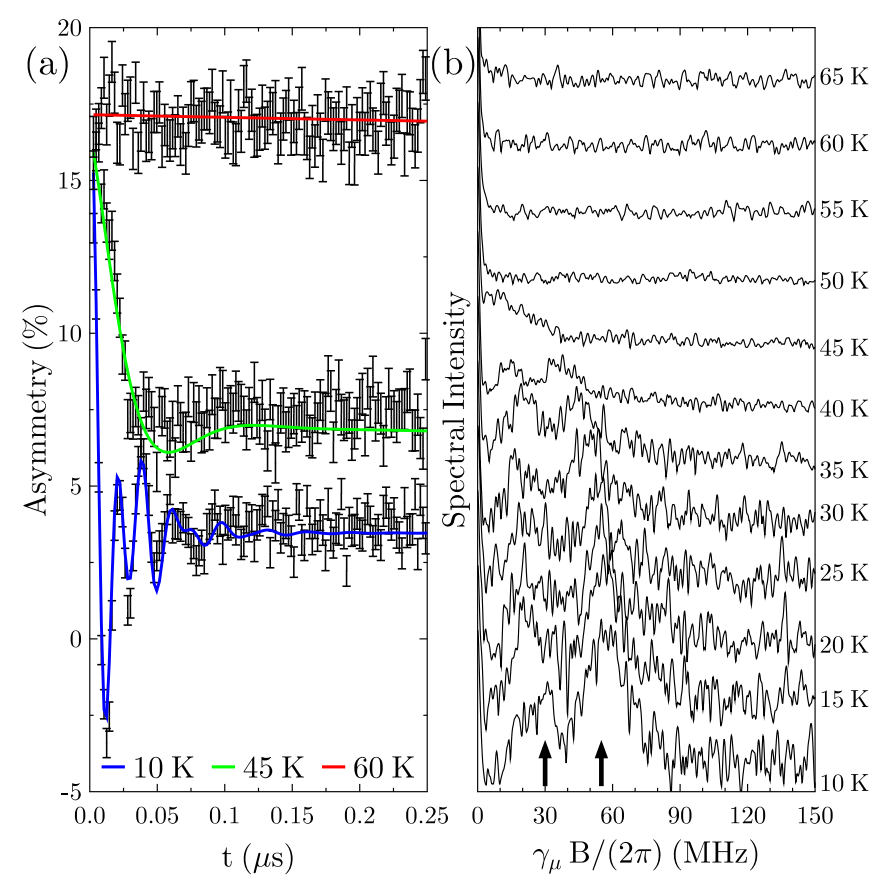

FIG. 5. (a) ZF- $\mu^{+} \mathrm{SR}$ asymmetry spectra below, near and above the transition in $\mathrm{Pb}_{2} \mathrm{CoOsO}_{6}$. The blue and green lines represent fits as in Eq. (1), the red line a Lorentzian relaxation fit of the form $A \exp (-\lambda t)$. (b) Fourier transform spectra of the first $0.5 \mu \mathrm{s}$ of the muon asymmetries (vertically shifted for readibility). The arrows indicate the position of the two frequencies at $10 \mathrm{~K}$.

equation

$$
A=A_{1} e^{-\lambda_{1} t} \cos \omega_{1} t+A_{2} e^{-\lambda_{2} t} \cos \omega_{2} t+A_{3} e^{-\lambda_{3} t} .
$$

The first two terms of Eq. (1) represent muons stopping in the sample and experiencing long-range magnetic order with precession angular frequencies $\omega_{i}=\gamma_{\mu} \mathrm{B}_{\mathrm{i}}$ and relaxation rates $\lambda_{i}$, where $\gamma_{\mu}=2 \pi \times 135.5 \mathrm{MHz} \mathrm{T}^{-1}$ is the muon gyromagnetic ratio and $\mathrm{B}_{\mathrm{i}}$ are the local fields. The third term accounts for muons stopping outside the sample, such as in the sample holder. The results for $\lambda_{i}$ and $v_{i}$ are plotted in Fig. 6, together with phenomenological order parameter models of the form $v=v_{0}\left[1-\left(T / T_{\mathrm{N}}\right)^{\alpha}\right]^{\beta}$ from which a critical temperature of $T_{\mathrm{N}}=45.5(10) \mathrm{K}$ can be extracted. Furthermore, the ratios of the amplitudes $A_{i}$ averaged over the measured temperatures below $T_{\mathrm{N}}$ indicate that about $35 \%$ of the muons in our experiments experienced the smaller magnetic field, about $60 \%$ the higher field, and about 5\% implanted outside the sample.

To compare the observed muon precession frequencies with ones predicted from different magnetic structures, it is necessary to establish the potential muon stopping sites in $\mathrm{Pb}_{2} \mathrm{CoOsO}_{6}$. To this end, we employed density-functional theory (DFT) calculations using the plane-wave program QUANTUM ESPRESSO [35] within the GGA [34]. We modeled the ions with ultrasoft pseudopotentials [36] and the muon with a norm-conserving hydrogen pseudopotential. The energy cutoffs for the wave function and the charge density were set to $80 \mathrm{Ry}$ and $800 \mathrm{Ry}$, respectively, and a $3 \times 3 \times 3$ MonkhorstPack $k$-space grid [37] was used for the integration over the Brillouin zone. Within these parameters, the calculations gave well-converged results and reproduced the experimentally

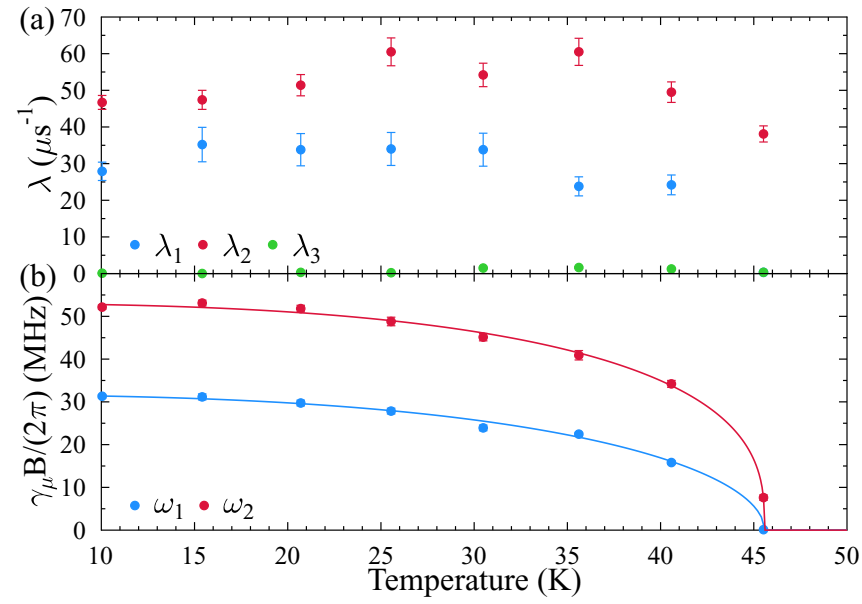

FIG. 6. Results of fitting Eq. (1) to the ZF- $\mu^{+}$SR assymetry spectra as a function of temperature. (a) Muon relaxation rates, (b) muon precession frequencies. Solid lines in (b) represent phenomenological order parameter fits of the form $v=v_{0}\left[1-\left(T / T_{\mathrm{N}}\right)^{\alpha}\right]^{\beta}$.

observed atomic positions and lattice parameters within a $1 \%$ accuracy. The results shown in Fig. 7 were visualized with the VESTA software [18].

We use the converged electron density to map out the electrostatic Coulomb potential of $\mathrm{Pb}_{2} \mathrm{CoOsO}_{6}$ throughout its unit cell, as plotted in Fig. 7. The global maximum is used as the reference point, since large values of the Coulomb potential correspond to a low energy cost to add a positive charge and such regions have been found to be a reliable first-order estimate of potential muon sites [38,39]. Additionally, we performed relaxation calculations, which allow for distortions of the lattice due to the implanted muon, which lead to muon site candidates in good agreement with the sites predicted by

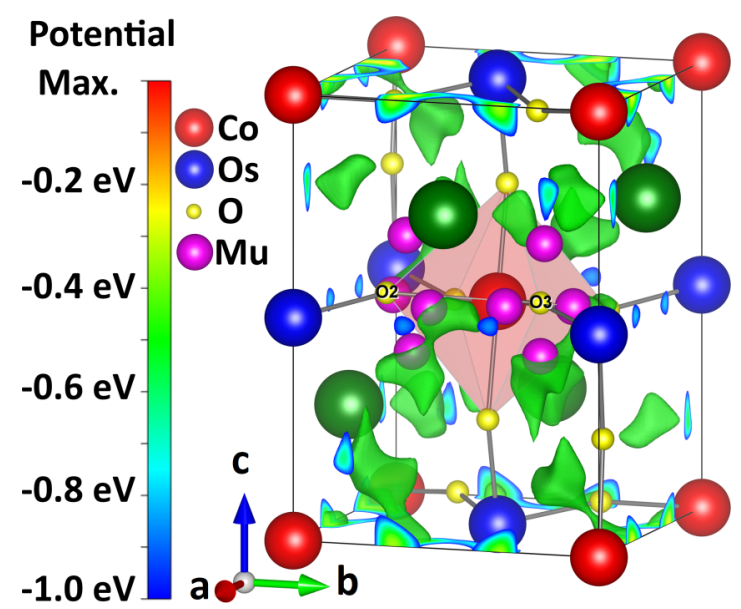

FIG. 7. Electrostatic Coulomb potential of $\mathrm{Pb}_{2} \mathrm{CoOsO}_{6}$ computed with DFT. The potential is shown on the surface of the unit cell up to $1.0 \mathrm{eV}$ below its maximum value, and a green isosurface is plotted within the unit cell at $0.5 \mathrm{eV}$ below the maximum. In addition, the eight muon site candidates (or a symmetry equivalent point) obtained from DFT relaxation calculations are also plotted in this undistorted unit cell to highlight the close symmetry relation between them. Note that the $\mathrm{Pb}$ ions are excluded for readability reasons. 
TABLE II. Summary of DFT $+\mu$ calculations. From left to right: Site label, fractional coordinates of muon site candidates from relaxation calculation, energy difference per unit cell for different muon site configurations, label of oxygen with which the muon forms an O-H-like $1.0 \AA$ bond.

\begin{tabular}{|c|c|c|c|c|c|}
\hline \multirow{2}{*}{$\begin{array}{l}\text { Site } \\
\text { Label }\end{array}$} & \multicolumn{3}{|c|}{ Relaxed position } & \multirow{2}{*}{$\begin{array}{c}\Delta \mathrm{E} / \mathrm{u} . \mathrm{c} . \\
(\mathrm{meV})\end{array}$} & \multirow{2}{*}{$\begin{array}{l}\mathrm{O}-\mathrm{H} \\
\text { with }\end{array}$} \\
\hline & $x$ & $y$ & $z$ & & \\
\hline Mu1 & 0.786 & 0.225 & 0.838 & 0 & $\mathrm{O} 3$ \\
\hline $\mathrm{Mu} 2$ & 0.264 & 0.720 & 0.334 & 4.5 & $\mathrm{O} 2$ \\
\hline Mu3 & 0.295 & 0.878 & 0.026 & 6.5 & $\mathrm{O} 3$ \\
\hline Mu4 & 0.878 & 0.699 & 0.983 & 24.5 & $\mathrm{O} 2$ \\
\hline Mu5 & 0.880 & 0.288 & 0.988 & 24.8 & $\mathrm{O} 3$ \\
\hline Mu6 & 0.305 & 0.121 & 0.015 & 40.5 & $\mathrm{O} 2$ \\
\hline $\mathrm{Mu} 7$ & 0.710 & 0.291 & 0.403 & 46.6 & $\mathrm{O} 2$ \\
\hline Mu8 & 0.710 & 0.711 & 0.407 & 46.8 & $\mathrm{O} 3$ \\
\hline
\end{tabular}

the local maxima of the electrostatic potential. In fact, we find that there are eight potential muon site candidates, Mu1-Mu8, which are all fairly closely related in energy and symmetry due to the proximity of the crystal structure of $\mathrm{Pb}_{2} \mathrm{CoOsO}_{6}$ to a more symmetric cubic one. These muon sites, plotted in Fig. 7 and tabulated in Table II, are also all characterized by an $\mathrm{O}-\mathrm{H}-\mathrm{like}$ bond between the muon and an oxygen with a bond length of about $1.0 \AA$, which is a typical occurrence in oxygen-containing compounds [38,39].

The muon asymmetry, plotted in Fig. 5(a) for three temperatures, exhibits an oscillatory beating pattern at low temperatures indicative of long-range magnetic ordering. The Fourier transform spectra of the ZF- $\mu^{+}$SR asymmetries, presented in Fig. 5(b), reveals two broad peaks centered around muon precession frequencies of roughly $30 \mathrm{MHz}$ and $55 \mathrm{MHz}$ at the lowest measured temperatures which vanish above the transition temperature.

With the potential muon spotting sites from Table II, we can calculate the local magnetic fields at each of these sites. Because our neutron experiments revealed an AFM ordering, the Lorentz and demagnetizing fields are zero and since the muon sites are far from the Os and Co ions we also expect hyperfine field contributions to be negligible, such that we only have to focus on the dipolar fields:

$$
\mathbf{B}_{\text {local }} \approx \mathbf{B}_{\mathrm{dip}}=\sum_{i} \frac{\mu_{o}}{4 \pi\left|\Delta \mathbf{r}_{i}\right|^{3}}\left[\frac{3\left(\mu_{i} \cdot \Delta \mathbf{r}_{i}\right) \Delta \mathbf{r}_{i}}{\left|\Delta \mathbf{r}_{i}\right|^{2}}-\boldsymbol{\mu}_{i}\right] .
$$

Here, the $\mathbf{r}_{i}$ correspond to the relative positions of the magnetic moments $\boldsymbol{\mu}_{i}$ with respect to the muon. Using the total moment size, as listed in Table I, we computed the local magnetic fields, assuming a fraction $x$ of the total moment lies on the Os sites, while a fraction $1-x$ of the total moment lies on the Co sites, for each of the muon site candidates and their 16 symmetry equivalent sites in the magnetic unit cell. The resulting muon precession frequencies are plotted in Fig. 8. Note that each line in Fig. 8 actually represents eight nearly identical lines, such that the 16 positions symmetry equivalent to the Mu1 site actually only lead to two distinct experimentally observable frequencies (unless the Os moment fraction $x$ is very close to 0 or 1 ). We can further note that the local fields at some of the muon stopping sites are almost identical,

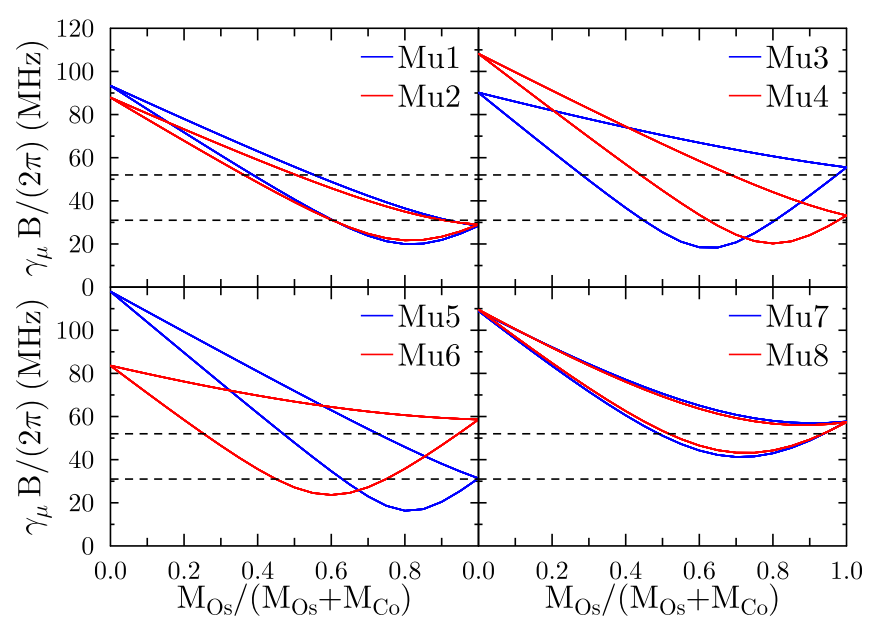

FIG. 8. Muon precession frequencies computed from the dipolar fields expected at the DFT-calculated muon sites. The magnetic structure is taken to be that shown in Fig. 3(b) of the main text, and the total moment size that in Table I. The fraction of the Os moment to the total moment was varied in line with the inset of Fig. 3(a). The muon site labels correspond to those in Table II. Note that the frequencies at all 16 symmetry-equivalent positions for each muon site are plotted, which results in pairs of eight very similarly and visually indistinguishable curves in each case.

owing to the approximate octahedral symmetry around each Co ion (see Fig. 7). Based on the muon precession frequencies shown in Fig. 8, we can identify two scenarios which can plausibly explain our experimentally observed frequencies: Either only the two energetically most favorable sites (Mu1 and Mu2) are significantly occupied and $x \approx 0.5$ or all eight of the identified muon site candidates might be occupied and $x \approx 1$. Both scenarios would lead to only two experimentally distinguishable frequencies, with a ratio of the oscillation amplitudes of these two frequencies expected in the region of $1: 1$, very roughly in line with that observed experimentally.

\section{DISCUSSION}

Of the two realistic possibilities outlined above, the scenario with $x \approx 1$ (i.e., the full moment is on the osmium site) is very unlikely. First, this scenario would require that the cobalt fails to order, contrary to other known double perovskites of mixed $3 d-5 d$ cations, in which the $3 d$ cation is either the only element that orders or the first to order by a significant margin. Second, the $\mathrm{Os}^{6+}$ in $\mathrm{Pb}_{2} \mathrm{CoOsO}_{6}$ would have to carry a moment (approximately $4 \mu_{\mathrm{B}}$ ) substantially greater than that which has been observed in any osmiumcontaining compound to date; and indeed greater than should be possible even for an ideal $J=2$ case (giving $3.6 \mu_{\mathrm{B}}$ ), which anyway does not seem to be favored for Os due to covalency effects that are substantial compared to $3 d$ and even $4 d$ elements [40-42]. The likelihood of such a large moment on Os is further diminished by recent results on other $5 d^{2}$ osmium double-perovskites $\mathrm{Ba}_{2} \mathrm{CaOsO}_{6}$ (ordered moment $<0.2 \mu_{\mathrm{B}}$ [42]), $\mathrm{Sr}_{2} \mathrm{CoOsO}_{6}$ (ordered moment $1.8 \mu_{\mathrm{B}}$ $[29,30]$ ), $\mathrm{Ca}_{3} \mathrm{OsO}_{6}$ (moment estimated from Curie-Weiss fit $<2 \mu_{\mathrm{B}}$ [43]), and both $\mathrm{Sr}_{2} \mathrm{MgOsO}_{6}$ and $\mathrm{Ca}_{2} \mathrm{MgOsO}_{6}$ (ordered moment $\approx 1.8 \mu_{\mathrm{B}}$ [44]). The weight of evidence 
overwhelmingly suggests that both the Os and Co ions carry an ordered moment in the magnetically ordered phase.

\section{CONCLUSION}

We conclude that the AFM phase transition in $\mathrm{Pb}_{2} \mathrm{CoOsO}_{6}$, involving the simultaneous magnetic order on both the $\mathrm{Co}$ and Os sublattices removes the center of crystal symmetry and through magnetoelastic coupling relaxes the structure into the polar multiferroic Shubnikov group $\mathrm{P}_{a} \mathrm{c}$. In $\mathrm{Pb}_{2} \mathrm{CoOsO}_{6}$, the spin-driven nature of the structural transition is reminiscent of a type-II hybrid improper multiferroic transition, in which magnetic order removes inversion symmetry via some higher order invariant in the free energy [11,12]. A key difference between the present case and all known multiferroics, however, is that the transition in $\mathrm{Pb}_{2} \mathrm{CoOsO}_{6}$ occurs entirely in the metallic state. Oxide NCSMs are still very rare and the materials known up to now exist because the polar displacements are almost entirely decoupled from the conduction electrons. The magnetic mechanism for acentricity in $\mathrm{Pb}_{2} \mathrm{CoOsO}_{6}$ does not suffer from this constraint and could provide a route for the discovery of other oxide NCSMs and their associated phenomena. Thus, the phase transition we have illustrated in $\mathrm{Pb}_{2} \mathrm{CoOsO}_{6}$ could provide a guiding principle for the future discovery of oxide NCSMs.

\section{ACKNOWLEDGMENTS}

The study in Japan was supported in part by JSPS KAKENHI Grants No. JP20H05276 and No. 19H05819, a research grant from Nippon Sheet Glass Foundation for Materials Science and Engineering (No. 40-37), and Innovative Science and Technology Initiative for Security (Grant No. JPJ004596), ATLA. The work in Oxford was supported by EPSRC Grant No. EP/N034872 and No. EP/N023803. H.M.W. acknowledges the support from NSF of China and the 973 program of China (Grants No. 2011CBA00108 and No. 2013CB921700). Y.F.G. acknowledges the support of Shanghai Pujiang Program, Grant No. 17PJ1406200. We performed the SXRD measurements with the approval of the NIMS beamline station (Proposal No. 2013B4503). The authors acknowledge the use of the University of Oxford Advanced Research Computing (ARC) facility [45].

A.J.P. and H.L.F. contributed equally to this work.
[1] V. P. Mineev and Y. Yoshioka, Phys. Rev. B 81, 094525 (2010).

[2] D. Puggioni and J. M. Rondinelli, Nat. Commun 5, 3432 (2014).

[3] S. Mühlbauer, B. Binz, F. Jonietz, C. Pfleiderer, A. R. A. Neubauer, R. Georgii, and P. Böni, Science 323, 915 (2009).

[4] S. Fujimoto, J. Phys. Soc. Jpn. 76, 051008 (2007).

[5] E. Bauer and M. Sigrist, Non-Centrosymmetric Superconductors: Introduction and Overview (Springer, Heidelberg, 2012).

[6] K. V. Samokhin, Ann. Phys. 359, 385 (2015).

[7] G. Dresselhaus, Phys. Rev. 100, 580 (1955).

[8] I. A. Sergienko, V. Keppens, M. McGuire, R. Jin, J. He, S. H. Curnoe, B. C. Sales, P. Blaha, D. J. Singh, K. Schwarz, and D. Mandrus, Phys. Rev. Lett. 92, 065501 (2004).

[9] Y. Shi, Y. Guo, X. Wang, A. J. Princep, D. Khalyavin, P. Manuel, Y. Michiue, A. Sato, K. Tsuda, S. Yu, M. Arai, Y. Shirako, M. Akaogi, N. Wang, K. Yamaura, and A. T. Boothroyd, Nat. Mater. 12, 1024 (2013).

[10] P. W. Anderson and E. I. Blount, Phys. Rev. Lett. 14, 217 (1965).

[11] N. A. Benedek and C. J. Fennie, Phys. Rev. Lett. 106, 107204 (2011).

[12] A. Stroppa, P. Barone, P. Jain, J. M. Perez-Mato, and S. Picozzi, Adv. Mater. 25, 2284 (2013).

[13] Y. Kanke, M. Akaishi, S. Yamaoka, and T. Taniguchi, Rev. Sci. Instrum. 73, 3268 (2002).

[14] T. Taniguchi, M. Akaishi, Y. Kanke, and S. Yamaoda, Rev. Sci. Instrum. 75, 1959 (2004).

[15] See Supplemental Material at http://link.aps.org/supplemental/ 10.1103/PhysRevB.102.104410 for details of synthesis methodology, $\mathrm{x}$-ray characterization, and bulk magnetic, thermal and transport measurements.

[16] M. Tanaka, Y. Katsuya, and A. Yamamoto, Rev. Sci. Instrum. 79, 075106 (2008).

[17] F. Izumi and T. Ikeda, Mater. Sci. Forum. 321-324, 198 (2000).

[18] K. Momma and F. Izumi, J. Appl. Cryst. 44, 1272 (2011).

[19] A. M. Glazer, Acta Cryst B 28, 3384 (1972).
[20] C. Ederer, T. Harris, and R. Kováčik, Phys. Rev. B 83, 054110 (2011).

[21] M. Azuma, K. Takata, T. Saito, S. Ishiwata, Y. Shimakawa, and M. Takano, J. Am. Chem. Soc. 127, 8889 (2005).

[22] M. Hoelzel, A. Senyshyn, N. Juenke, H. Boysen, W. Schmahl, and H. Fuess, Nucl. Instrum. Methods Phys. Res., A 667, 32 (2012).

[23] L. C. Chapon, P. Manuel, P. G. Radaelli, C. Benson, L. Perrott, S. Ansell, N. J. Rhodes, D. Raspino, D. Duxbury, E. Spill, and J. Norris, Neutron News 22, 22 (2011).

[24] J. Rodriguez-Carvajal, Physica B 192, 55 (1993); FullProf Suite, http://www.ill.eu/sites/fullprof/.

[25] K. Kobayashi, T. Nagao, and M. Ito, Acta Cryst. A 67, 473 (1991).

[26] S. C. Miller and W. F. Love, Tables of Irreducible Representations of Space Groups and Co-Representations of Magnetic Space Groups (Pruett, Boulder, 1967).

[27] H. C. Walker, F. Fabrizi, L. Paolasini, F. de Bergevin, J. HerreroMartin, A. T. Boothroyd, D. Prabhakaran, and D. F. McMorrow, Science 333, 1273 (2011).

[28] A. K. Paul, M. Reehuis, V. Ksenofontov, B. Yan, A. Hoser, D. M. Többens, P. M. Abdala, P. Adler, M. Jansen, and C. Felser, Phys. Rev. Lett. 111, 167205 (2013).

[29] R. Morrow, R. Mishra, O. D. Restrepo, M. R. Ball, W. Windl, S. Wurmehl, U. Stockert, B. Büchner, and P. M. Woodward, J. Am. Chem. Soc. 135, 18824 (2013).

[30] B. Yan, A. K. Paul, S. Kanungo, M. Reehuis, A. Hoser, D. M. Többens, W. Schnelle, R. C. Williams, T. Lancaster, F. Xiao, J. S. Möller, S. J. Blundell, W. Hayes, C. Felser, and M. Jansen, Phys. Rev. Lett. 112, 147202 (2014).

[31] Isotropy software suite, http://iso.byu.edu.

[32] K. I. Kugel and D. I. Khomskii, Sov. Phys. Usp. 25, 231 (1982).

[33] Open source package for material explorer, http://www. openmx-square.org. 
[34] J. P. Perdew, K. Burke, and M. Ernzerhof, Phys. Rev. Lett. 77, 3865 (1996).

[35] P. Gianozzi et al., J. Phys.: Condens. Matter 21, 395502 (2009).

[36] A. M. Rappe, K. M. Rabe, E. Kaxiras, and J. D. Joannopoulos, Phys. Rev. B 41, 1227 (1990).

[37] H. J. Monkhorst and J. D. Pack, Phys. Rev. B 13, 5188 (1976).

[38] J. S. Möller, P. Bonfà, D. Ceresoli, F. Bernardini, S. J. Blundell, T. Lancaster, R. De Renzi, N. Marzari, I. Watanabe, S. Sulaiman, and M. I. Mohamed-Ibrahim, Phys. Scr. 88, 068510 (2013).

[39] F. R. Foronda, F. Lang, J. S. Möller, T. Lancaster, A. T. Boothroyd, F. L. Pratt, S. R. Giblin, D. Prabhakaran, and S. J. Blundell, Phys. Rev. Lett. 114, 017602 (2015).
[40] S. Gangopadhyay and W. E. Pickett, Phys. Rev. B 91, 045133 (2015).

[41] A. E. Taylor, R. Morrow, D. J. Singh, S. Calder, M. D. Lumsden, P. M. Woodward, and A. D. Christianson, Phys. Rev. B 91, 100406(R) (2015).

[42] C. M. Thompson, J. P. Carlo, R. Flacau, T. Aharen, I. A. Leahy, J. R. Pollichemi, T. J. S. Munsie, T. Medina, G. M. Luke, and J. Munevar, J. Phys.: Condens. Matter 26, 306003 (2014).

[43] H. L. Feng, Y. Shi, Y. Guo, J. Li, A. Sato, Y. Sun, X. Wang, S. Yu, C. I. Sathisha, and K. Yamaura, J. Solid State Chem. 201, 186 (2013).

[44] Y. Yuan, H. L. Feng, M. P. Ghimire, Y. Matsushita, Y. Tsujimoto, J. He, M. Tanaka, Y. Katsuya, and K. Yamaura, Inorg. Chem. 54, 3422 (2015).

[45] http://dx.doi.org/10.5281/zenodo.22558. 